\title{
Variability in Measurement of Allergen Skin Testing Results among Allergy-Immunology Specialists
}

Kenny Y C Kwong*, Tiffany Jean and Nasser Redjal

Division of Allergy-Immunology, Department of Pediatrics, Harbor-UCLA Medical Center, USA

\begin{abstract}
Epicutaneous skin testing for sensitization to aero-allergens in patients with allergic diseases requires measurement of skin wheal and erythema in response to antigens. This may be variable between different providers especially among patients with dark skin pigmentation. Purpose of this study is to determine whether Allergy-Immunology specialists vary in their measurement of epicutaneous skin tests in patients with differential skin pigmentation. 3 patients with differing skin tones were skin tested using various concentrations of histamine to produce increasing sizes of skin wheal and erythema reaction. High quality photographs of these were taken and sent to 20 board certified/eligible Allergy-Immunologists to measure the wheal and erythema size. There was poor agreement among specialists in measurement of both wheal and flare sizes from patient with darkest skin tone. In contrast in patients with lighter skin tones there was fair to good agreement and excellent agreement from specialists in measurement of wheal and flare sizes respectively. Measurement of skin test results in patients with dark skin pigmentation may have significant variability even among specialists and in vitro tests may be more appropriate in these patients.
\end{abstract}

Keywords: Allergen skin; Pigmentation; Epicutaneous skin tests

\section{Introduction}

Epicutaneous skin testing is recommended by National AllergyImmunology guidelines as the initial preferred method to test for environmental and food allergens in sensitized patients. The tests are safe, relatively inexpensive and provide results as soon as 20 minutes after administration [1]. There are certain conditions in which skin testing is accepted as unreliable. These include underlying skin conditions such as atopic dermatitis and medications which reduce cutaneous reactivity to antigens including anti-histamines and tricyclic anti-depressants [1,2]. Other variables also can affect results of skin testing although in less definitive and more variable fashion. Skin reactivity has been shown to change with age, quality of testing reagents, testing device and different parts of the body where testing took place [1,3-6]. Finally an accepted but less studied variable is variability in provider interpretation of skin tests particularly in patients with varying degrees of skin pigmentation [1]. Under these conditions use of in vitro allergy tests such as in vitro serum specific allergen IgE may be more accurate and reliable.

Skin testing results are usually interpreted by measuring the size of the wheal and flare in reaction to epidermal deposition of antigens. In the past significant number of Allergy providers utilized a semiqualitative scoring system $(0$ to +4$)$ to interpret skin test results $[1,2]$. The American Academy of Asthma Allergy and Immunology (AAAAI) in the most current practice parameter on diagnostic testing has recommended against using this method and to measure both wheal and flare size of skin testing, presenting the result as the mean of the horizontal and orthogonal measurements [1]. McCann and Ownby have shown that there is considerable variability in reading skin tests using the semi-qualitative method even among board certified/eligible allergist [2]. However to the best of our knowledge there have been no studies to determine if there is significant variability in measuring skin test results using mean horizontal/orthogonal method among allergyimmunology specialists; and whether differences in skin pigmentation or tone affects any variability. This study endeavors to see whether allergy-immunology specialists show variability in measuring skin tests using this method and whether there is greater variability in these results in patients with darker compared to lighter skin pigmentation.

\section{Methods}

\section{Skin tests}

3 subjects with differing skin tone grades according to the Fitzpatrick scale underwent epicutaneous skin testing after informed consent was obtained. 8 individual prick/puncture tests were performed on the forearms of each subject using a commercially available 8 pronged multitest device (Lincoln diagnostics, Decatus IL). Each subject was tested with histamine solution at full commercial strength and dilutions of 1:10, 1:100, 1:1000, 1:10000, 1: 100000, 1:000000 and a negative commercial skin test control of glycerolsaline (HollisterStier, Spokane WA). Digital photographs were taken of each individual's skin test 20 minutes after application using an 8 megapixel digital camera. Photographs were printed on high quality glossy 8 "X10" photo quality paper using a commercial photography laboratory (Fromex, Long Beach CA). The ratio of the skin tests on the photograph to actual size of in vivo skin tests was 1:1. Skin tests were labeled A through $\mathrm{H}$. These photographs were sent to 30 board certified/eligble allergist-immunologists practicing in Southern California. Instructions were given to measure each skin test (measuring the wheal and flare) according to the AAAAI 2008 practice parameters for diagnostic allergy testing [1]. This was the (horizontal length of the wheal or flare

*Corresponding author: Kenny Yat-Choi Kwong, Division of Allergy-Immunology, Department of Pediatrics, Harbor-UCLA Medical Center, 1000 West Carson Street N-25, Torrance California 90509, USA, Tel: 310-222-4162; Fax: 310-222-4006; E-mail: kkwongusc@yahoo.com

Received December 17, 2013; Accepted January 28, 2014; Published February 02,2014

Citation: Kwong KYC, Jean T, Redjal N (2014) Variability in Measurement of Allergen Skin Testing Results among Allergy-Immunology Specialists. J Allergy Ther 5: 160. doi:10.4172/2155-6121.1000160

Copyright: (c) 2014 Kwong KYC, et al. This is an open-access article distributed under the terms of the Creative Commons Attribution License, which permits unrestricted use, distribution, and reproduction in any medium, provided the original author and source are credited. 
Citation: Kwong KYC, Jean T, Redjal N (2014) Variability in Measurement of Allergen Skin Testing Results among Allergy-Immunology Specialists. J Allergy Ther 5: 160. doi:10.4172/2155-6121.1000160

Page 2 of 5

+orthogonal length of the wheal or flare) divided by 2 (measured in millimeters). An honorarium of $\$ 100$ US was offered for participation in the study.

The primary outcome was the reliability or agreement of each wheal and flare skin test reaction measured by 20 individual specialists. This intra-class correlation (ICC) was calculated as described by Shrout and Fleiss [7].

The study was reviewed and approved by the institutional review board of the Los Angeles Biomedical Foundation.

\section{Results}

The subjects tested had differential skin pigmentation. All subjects also had seasonal and perennial allergic rhinitis with sensitization to various perennial and seasonal allergens (data not shown). All subjects reacted to epicutaneous histamine testing with wheal and flare responses in increasing sizes to increasing concentrations of histamine. Photographs of wheal and flare reactions were more distinct on subject with dark (Figures 1a and 2a) compared to subject lighter skin tones (Figure 3a).

20 surveys were returned and results recorded completely for analysis. For both the wheal and flare reactions there was variability in measurements by respondents. In general there was variability between respondents in measurements of both wheal and flare reactions with more variability in measurement of the wheals compared to flares in all patients and more variability in both wheal and flare measurements in patient with darkest compared to ones with lighter skin pigmentations (Figures 1b,2b,3b).

Overall, across the 3 types of skin tones there was excellent agreement among specialists in measurement of the flare (ICC $=0.834)$ compared to fair to good agreement in wheal measurement (ICC $=0.464)$. Agreement amongst specialists in measurement of the flare was excellent in patient with lighter skin tones (ICC $=0.792$ and 0.902 ) but poor in patient with darkest skin tone (ICC=0.060). Agreement amongst specialists in measurement of the wheal was fair to good in patient with lighter skin tones (ICC $=0.579$ and 0.657 ) but poor in patient with darkest skin tone $(\mathrm{ICC}=0.067)$ (Table 1$)$. In general there was less variability in measurement of both wheal and flare sizes among specialists when the reactions were larger in size across the different skin tones although there was no statistically significant difference.

\section{Discussion}

In this study we demonstrated that there was significant variability in measurement of skin test wheal and flare reactions in patients with dark skin pigmentation among allergy specialists. Agreement between

\section{a)}
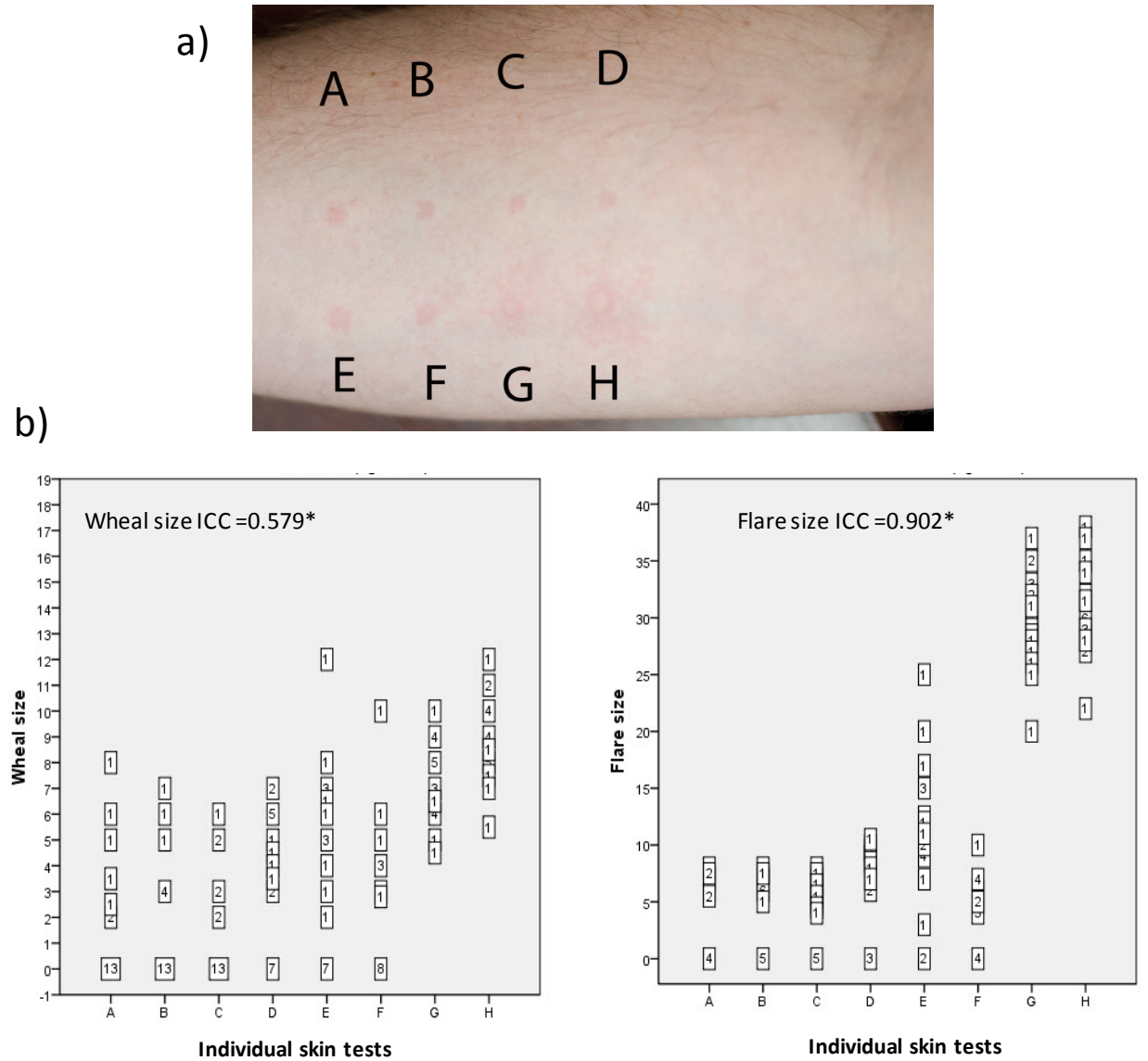

Figure 1: a) Photograph of skin wheal and flare reactions on inner forearm of subject with light skin tone. *ICC-Intraclass correlation. Correlation of observed wheal and flare measurements across raters (specialists) within the same patients b) Distribution of wheal and flare measurements by 20 allergy specialists of 8 skin wheal and flare skin results. Interpretation example: For skin test A wheal reaction, 13 specialists rated the wheal size a $0 \mathrm{~mm}, 2$ rated size as $2 \mathrm{~mm}, 1$ rated size as $2.5 \mathrm{~mm}, 1$ rated size as $3.5 \mathrm{~mm}$, 1 rated size as $5 \mathrm{~mm}, 1$ rated size as $6 \mathrm{~mm}$ and 1 rated size as $8 \mathrm{~mm}$. 
a)

b)
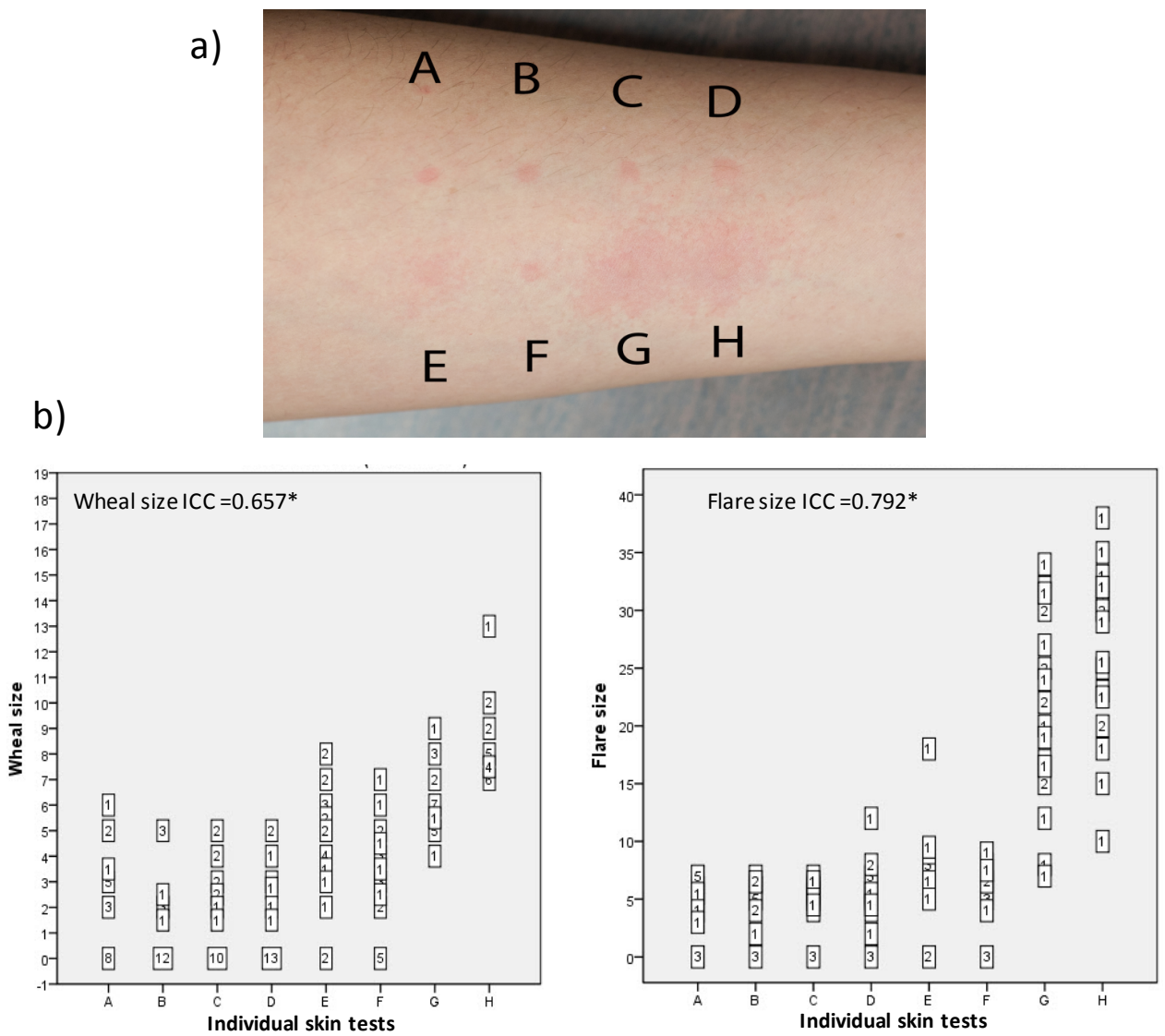

Figure 2: a) Photograph of skin wheal and flare reactions on inner forearm of subject with light skin tone. *ICC-Intraclass correlation. Correlation of observed wheal and flare measurements across raters (specialists) within the same patients b) Distribution of wheal and flare measurements by 20 allergy specialists of 8 skin wheal and flare skin results. See figure 1 for Interpretation example.

\begin{tabular}{|l|l|l|}
\hline & Wheal & Flare \\
\hline Group & ICC $^{*}$ & ICC $^{*}$ \\
\hline Overall & 0.464 & 0.834 \\
\hline Photo A (Lightest tone) & 0.579 & 0.902 \\
\hline Photo B (Medium tone) & 0.657 & 0.792 \\
\hline Photo C (Dark tone) & 0.067 & 0.060 \\
\hline
\end{tabular}

*ICC: Intraclass correlation. Correlation of observed wheal and flare measurements across raters (specialists) within the same patients

Table 1: Reliability (Shrout-Fleiss) of allergy specialists' assessment of wheal and flare size in patients with differing skin tones.

specialists in measurements of wheal and flare reactions in fairer skinned patients was fair to good and excellent respectively.

Allergen skin testing is cost effective, safe, simple to administer and results available within 20 to 30 minutes [1]. Multiple variables however affect the accuracy and reproducibility of skin test results. Well documented variables include concomitant use of medications that influence effects of mast cell mediators including anti-histamines and mast cell stabilizers; Diseases which affect the integrity of the skin such as atopic dermatitis; And age of the patients wherein infants and young children have less skin reactivity [1,4-6]. Skin pigmentation or tone has been cited by practice guidelines as one variable which may affect skin test accuracy and reproducibility [1]. While this is logical there are few studies supporting this. To the best of our knowledge this is the first study which shows that there is significant variability in measurement of skin test wheal and flare sizes in patients with dark skin tones. This suggests that interpretation of allergen skin testing may be problematic in these patients.

In this study allergy specialists are able to measure flare more consistently than wheal reactions. While not statistically significant there was more measurement variability when wheal sizes were smaller in magnitude. Most providers measure both wheal and flare sizes in determination of allergic sensitization however some investigators advocate the importance of the wheal $[7,8]$. Current National allergic testing guidelines recommend a minimum of $3 \mathrm{~mm}$ wheal reaction for a positive test $[1,9-14]$. Wheal size to various environmental antigens more than $4 \mathrm{~mm}$ is predictive of development of allergic disease $[1,15]$. Likewise wheal size of more than $8 \mathrm{~mm}$ to milk, egg and peanut are highly predictive of clinical reactivity in young children with food allergy $[1,16,17]$. There is however little data on sensitivity, specificity and predictive value of the flare response. Variability of providers to consistently measure wheal size may result in inaccurate interpretation of test results particularly when wheal sizes are smaller in magnitude. Of concern is variability with wheal sizes in the $3 \mathrm{~mm}$ range which could inaccurately result in false positive or negative tests.

Interpretations made by this study need to be taken with caution due to several limitations. Measurements of skin reactions are were made from photographic images and not in vivo. Wheal reactions are three dimensional and the ability to palpate the borders of the wheal will increase the accuracy of the ability of specialists to more accurately measure the test results. Further ability to rotate the arm and varying 
Citation: Kwong KYC, Jean T, Redjal N (2014) Variability in Measurement of Allergen Skin Testing Results among Allergy-Immunology Specialists. J Allergy Ther 5: 160. doi:10.4172/2155-6121.1000160

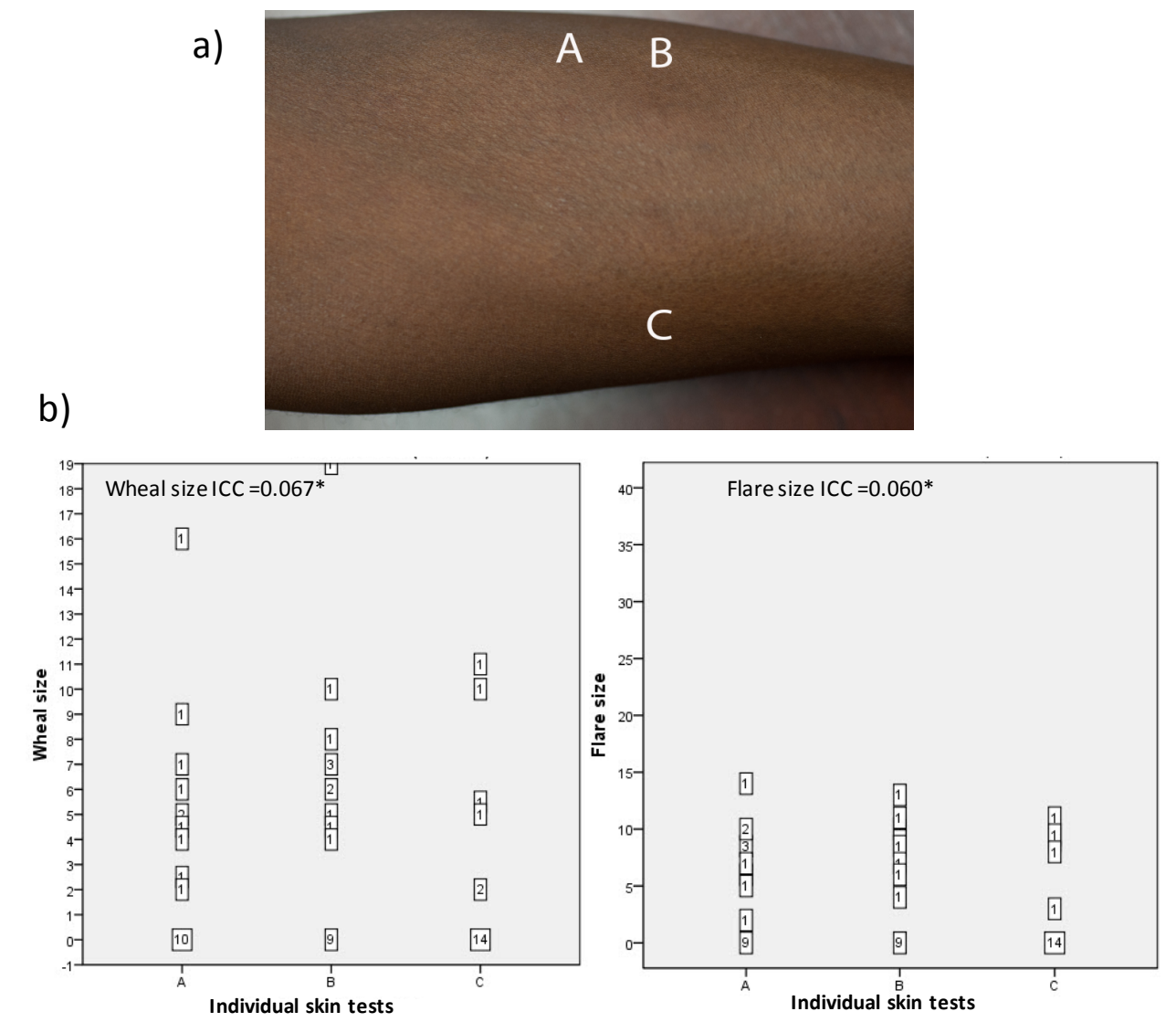

Figure 3: a) Photograph of skin wheal and flare reactions on inner forearm of subject with light skin tone. *ICC-Intraclass correlation. Correlation of observed wheal and flare measurements across raters (specialists) within the same patients b) Distribution of wheal and flare measurements by 20 allergy specialists of 3 skin wheal and flare skin results. See figure 1 for Interpretation example.

the direction of the light source increases the ability of the provider to better discriminate the border of both wheal and flare reactions. The skin tones were subjectively chosen by the investigators to represent a range of skin pigmentation without the use of a standardized scale. There is likely more skin tones on both extreme spectrums of light and dark skin tones presented in this study. Agreement among specialists in measurement of wheal and flare reactions in these subjects may be different from present results. Increasing concentrations of histamine was used to elicit skin reactions and although histamine is used routinely as a positive control in allergen skin testing results may be different if actual skin testing allergen was used (along with in vitro specific IgE to respective allergen as control). Finally the sample size of both the skin tests and respondents is small and results may be different with larger sample sizes.

For over a century and still today, skin testing has been the recommended method to determine allergen sensitization in patients with allergic diseases $[1,18,19]$. Results of these tests are frequently used to determine allergy therapy. These may be expensive, have potential for serious adverse events and require significant commitment by patients and providers. Environmental controls may require purchase of expensive dust mite proof covers, removal of carpeting and purchase of HEPA air filters [20]. Domestic pets may have to be removed from households which may cause stress to patients [21]. Allergen immunotherapy recommended on basis of skin tests may result in systemic reactions and in rare cases death. Commitment to immunotherapy requires frequent injections or sublingual antigen administration for up to 5 years. Correct identification of an offending allergen requires positive allergy test results that are history consistent with symptoms elicited by the particular allergen [22]. Therefore allergy testing must be accurate. If skin testing has questionable accuracy in patients with dark skin tones then substitute tests with are not affected by skin pigmentation such as in vitro IgE tests may be better alternatives in these patients to test for allergen sensitization.

In conclusion, there may be significant variability in measurement of skin test reaction in patients with dark skin pigmentation/skin tones by Allergy-Immunology specialists. Care should be taken when interpreting skin test results in these patients and alternative allergy tests unaffected by skin tones be considered.

\section{Acknowledgements}

The authors wish to Thank Johana Raygoza for assistance with this study.

\section{Funding}

This study was funded by Thermo-Fisher Scientific.

\section{Conflicts of Interest}

KY Kwong has received grant support and serves on the speaker bureau from Thermo-Fisher Scientific. T Jean and N Redjal report no conflicts of interests.

\section{References}

1. Bernstein IL, Li JT, Bernstein DI, Hamilton R, Spector SL, et al. (2008) Allergy diagnostic testing: an updated practice parameter. Ann Allergy Asthma Immunol 100: S1-148.

2. McCann WA, Ownby DR (2002) The reproducibility of the allergy skin test 
Citation: Kwong KYC, Jean T, Redjal N (2014) Variability in Measurement of Allergen Skin Testing Results among Allergy-Immunology Specialists. J Allergy Ther 5: 160. doi:10.4172/2155-6121.1000160

Page 5 of 5

scoring and interpretation by board-certified/board-eligible allergists. Ann Allergy Asthma Immunol 89: 368-371.

3. Nelson HS (1983) Diagnostic procedures in allergy. I. Allergy skin testing. Ann Allergy 51: 411-418.

4. Ménardo JL, Bousquet J, Rodière M, Astruc J, Michel FB (1985) Skin test reactivity in infancy. J Allergy Clin Immunol 75: 646-651.

5. Campo P, Kalra HK, Levin L, Reponen T, Olds R, et al. (2006) Influence of dog ownership and high endotoxin on wheezing and atopy during infancy. J Allergy Clin Immunol 118: 1271-1278.

6. Barbee RA, Brown WG, Kaltenborn W, Halonen M (1981) Allergen skin-test reactivity in a community population sample: correlation with age, histamine skin reactions and total serum immunoglobulin E. J Allergy Clin Immunol 68: 15-19.

7. Shrout PE, Fleiss JL (1979) Intraclass correlations: uses in assessing rater reliability. Psychol Bull 86: 420-428.

8. Aas K (1980) Some variables in skin prick testing. Allergy 35: 250-252.

9. Nelson HS, Lahr J, Buchmeier A, McCormick D (1998) Evaluation of devices for skin prick testing. J Allergy Clin Immunol 101: 153-156.

10. Oppenheimer J, Nelson HS (2006) Skin testing: a survey of allergists. Ann Allergy Asthma Immunol 96: 19-23.

11. (1989) Skin tests used in type I allergy testing Position paper. Sub-Committee on Skin Tests of the European Academy of Allergology and Clinical Immunology. Allergy 44: 1-59.

12. Berkowitz RB, Tinkelman DG, Lutz C, Crummie A, Smith K (1992) Evaluation of the Multi-Test device for immediate hypersensitivity skin testing. J Allergy Clin Immunol 90: 979-985.

13. Koller DY, Pirker C, Jarisch R, Götz M (1992) Influence of the histamine control on skin reactivity in skin testing. Allergy 47: 58-59.

14. Björkstén F, Haahtela T, Backman A, Suoniemi I (1984) Assay of the biologic activity of allergen skin test preparations. J Allergy Clin Immunol 73: 324-331.

15. Bodtger U, Poulsen LK, Malling HJ (2003) Asymptomatic skin sensitization to birch predicts later development of birch pollen allergy in adults: a 3-year follow-up study. J Allergy Clin Immunol 111: 149-154.

16. Saarinen KM, Suomalainen H, Savilahti E (2001) Diagnostic value of skin-prick and patch tests and serum eosinophil cationic protein and cow's milk-specific IgE in infants with cow's milk allergy. Clin Exp Allergy 31: 423-429.

17. Kagan R, Hayami D, Joseph L, St Pierre Y, Clarke AE (2003) The predictive value of a positive prick skin test to peanut in atopic, peanut-naïve children. Ann Allergy Asthma Immunol 90: 640-645.

18. Taylor G, Walker J (1973) Charles Harrison Blackley, 1820-1900. Clin Allergy 3: 103-108.

19. Feinberg SM (1946) Allergy in Practice. (2ndedn) Chicago, IL: Year Book Medical Publishers: (IIb).

20. Blaiss MS (2010) Allergic rhinitis: Direct and indirect costs. Allergy Asthma Proc 31: $375-380$.

21. Ling M, Long AA (2010) Pet dander and difficult-to-control asthma: Therapeutic options. Allergy Asthma Proc 31: 385-391.

22. Cox L, Nelson H, Lockey R, Calabria C, Chacko T, et al. (2011) Allergen immunotherapy: a practice parameter third update. J Allergy Clin Immunol 127: S1-55. 